\title{
SITUAÇÃO EPIDEMIOLÓGICA E DIFUSÃO DA COVID-19 PELA REDE URBANA EM MINAS GERAIS, BRASIL
}

\section{EPIDEMIOLOGICAL SITUATION AND DIFFUSION OF COVID-19 THROUGH THE URBAN NETWORK IN MINAS GERAIS, BRAZIL}

\author{
Samuel do Carmo Lima \\ LAGEM/Universidade Federal de Uberlândia \\ samuel@ufu.br \\ Elivelton da Silva Fonseca \\ LAGEM/Universidade Federal de Uberlândia \\ elivelton.fonseca@gmail.com \\ Flavia de Oliveira Santos \\ LAGEM/Universidade Federal de Uberlândia \\ flavia.santos@ufu.br
}

\begin{abstract}
RESUMO
O Estado de Minas Gerais apresenta, diferentemente da maioria dos Estados brasileiros, um patamar de transmissão ainda baixo, porque suas principais cidades, Belo Horizontes, Uberlândia, Juiz de Fora, Contagem, Montes Claros não atingiram ainda o nível de transmissão da doença que pode colocar o Sistema de Saúde Pública em colapso, por falta de leitos de UTI, respiradores e equipamentos de proteção individual para os profissionais de saúde, como já se vê em Manaus, Belém, São Luiz, Fortaleza, Rio de Janeiro e São Paulo. O que se percebe é que, no início, a entrada do novo coronavírus no Estado seguiu o mesmo ocorrido nos demais Estados, desembarcando de avião na capital e nas cidades que tinham maior conectividade com a Europa, principalmente com a Itália, que era o epicentro da pandemia mundial nos meses de fevereiro e março de 2020. No início, a pandemia no Estado se manteve em níveis baixos porque as medidas de isolamento social foram suficientes para achatar a curva de transmissão. Mas, logo veio o crescimento da curva e o número de casos cresceu, porque o isolamento social foi enfraquecido pelos decretos municipais que flexibilizavam a quarentena e abria o comércio, mandando as pessoas para a rua. O momento agora é de apreensão, com o número de casos e óbitos por Covid-19 crescendo na capital e nas cidades médias polos regionais. No memento, o novo coronavírus está viajando de ônibus e de carro, das cidades polos regionais para as pequenas cidades do interior. A intensificação da transmissão nessas cidades pode ser catastrófica, porque nelas só tem a atenção básica à saúde, conforme diretrizes do SUS e elas se valem do sistema hospitalar de atenção secundária e terciária das cidades médias, que já estão chegando no limite de sua capacidade de leitos de UTI. Este trabalho tem por objetivo avaliar a situação epidemiológica e a Covid-19 no Estado de Minas Gerais, considerando a sua difusão pela rede urbana do Estado, a partir das cidades polos regionais.
\end{abstract}

Palavras chaves: COVID-19. Rede urbana. Risco. Vulnerabilidade. Contexto.

\begin{abstract}
The spread of the Covid-19 pandemic across the world was fast, reaching Europe, collapsing the health systems in Italy, Spain, France and the United Kingdom and then taking on unimaginable proportions in the USA. In Brazil, the State of Minas Gerais has been considered a low level of transmission, unlike most Brazilian states, because its main cities, Belo Horizonte, Uberlândia, Juiz de Fora, Contagem, Montes Claros have not yet reached the level of disease transmission that can collapse the Public Health System. Other states have seen the lack of ICU beds, mechanic ventilators and individual protective equipment for health professionals (e.g Manaus, Belém, São Luiz, Fortaleza, Rio de Janeiro and São Paulo). What can be seen is that, at the beginning, the entry of the new coronavirus in the State followed the same as in the other States, disembarking by plane in the capital and in the cities that had greater connectivity with Europe. Between February
\end{abstract}

Recebido em: 10/05/2020

Aceito para publicação em: 02/06/2020. 
and March, as Italy was the epicenter of the world pandemic, still direct flights coming from the country to Belo Horizonte might affect the spread in Minas Gerais. At first, the state's pandemic remained at low levels because measures of social isolation were sufficient to flatten the transmission curve. But, soon the growth of the curve was noticed due the weakening of social distancing, quarentine and isaolation by the municipal decrees, allowing circulation of people. The moment is now of apprehension in Minas Gerais, with the number of cases and deaths by Covid-19 growing in the capital city and in the medium-sized cities and regional poles. The new coronavirus is traveling by bus and car, from regional pole cities to small towns. The intensification of transmission in these cities can be catastrophic, because they are equiped only with primary health care, according to United Health System (SUS) guidelines. To make use of the secondary and tertiary hospital care system the population have to travel to the near medium-sized cities, which are already reaching the limit of their capacity to ICU beds. This work aim at the evaluation of the epidemiological situation and Covid-19 in the State of Minas Gerais, considering its diffusion through the urban network of the State, starting from the regional pole cities.

Keywords: COVID-19. Urban network. Risk. Vulnerability. Context.

\section{INTRODUÇÃO}

O mundo ainda está perplexo diante da pandemia, porque se trata de um problema que atinge o mundo todo, quase ao mesmo tempo, e pouco se pode fazer para evitá-la, a não ser restringir o contato social, com a quarentena, em que as pessoas permanecem isoladas em casa e, também, reduzir os deslocamentos entre as cidades e entre os países, porque é por aí que o vírus viaja de um país ao outro, de uma cidade a outra. Os remédios que existem no mercado farmacêutico não são totalmente eficazes para combater o vírus, não há vacina. $O$ vírus tem alta transmissibilidade e uma letalidade maior que a dos vírus que provocam as gripes sazonais (OPAS BRASIL, 2020).

A COVID-19 é uma doença emergente, causada pelo novo coronavírus (SARS-CoV-2), descoberta em Wuhan, uma cidade de cerca de 11 milhões de habitantes, na província de Hubei, na região central da China. Os quatro primeiros casos foram relatados em 29/12/2019, todos relacionados a um mercado de frutos do mar, que também comercializava animais silvestres, que são indicados como fonte da pandemia que se alastrou por todo o mundo. A doença foi identificada em 07/01/2020 e tem como principais sintomas febre, tosse, cansaço, dificuldades para respirar, provocando Síndrome Respiratória Aguda Grave (LI et al. 2020).

Em 13/01/2020, na Tailândia, foi notificado o primeiro caso de Covid-19 fora da China. Em 30/01/2020, a Organização Mundial da Saúde (OMS) declarou Emergência de Saúde Pública de Importância Internacional em razão do surto do novo coronavírus em Wuhan. Em 31/12/2019, a China notificou a OMS 44 casos de Covid-19. Em 11/01/2020, foi registrado o primeiro óbito por Covid-19 em Wuhan e, nesta dada, a OMS declarou estado de pandemia, quando a doença já havia alcançado 114 países, com 118 mil casos confirmados e 4.200 óbitos (OPAS BRASIL, 2020, BRASIL, 2020).

A disseminação da pandemia de Covid-19 pelo mundo foi rápida, chegando à Europa, provocando colapso dos sistemas de saúde na Itália, na Espanha, na França, no Reino Unido, e em seguida tomando grandes proporções nos EUA. Nestes países, nos meses de março e abril de 2020 , foram decretadas medidas de isolamento social, com quarentenas mais rigorosas e lockdowns, em determinados momentos e em determinadas regiões.

No Brasil, o primeiro caso de Covid-19 foi notificado em São Paulo, no dia 25/02/2020, cerca de dois meses após os primeiros casos terem surgido em Wuhan, na China. O paciente era um homem de 61 anos que havia chegado da Itália no dia 21/02/2020, com sintomas de febre, tosse seca, dor de garganta e coriza. Em 10/05/2020, já havia 162.699 pessoas contaminadas com o novo coronavírus e 11.123 óbitos por Covid-19 confirmados em diversas regiões do país (BRASIL, 2020).

Em Minas Gerais, o primeiro caso de Covid-19 foi confirmado em 08/03/2020. Era um paciente do sexo feminino, 47 anos, residente no município Divinópolis, com histórico de viagem para Itália, e retorno ao Brasil em 02/03/2020. A partir de 05/03/2020, apresentou sintomas leves de mialgia e coriza, sem necessidade de hospitalização. 
O objetivo deste trabalho é avaliar o contexto da difusão da Covid-19 pela rede urbana de Minas Gerais, considerando as conectividades entre a capital e as cidades polos regionais e destas com as pequenas cidades de sua região. Os conceitos básicos que nortearam as análises foram rede urbana, risco, vulnerabilidade e contexto.

\section{SITUAÇÃO EPIDEMIOLÓGICA DA COVID-19 EM MINAS GERAIS}

O Estado de Minas Gerais possui 853 municípios. Em 07/04/2020, em 549 municípios (76,1\%) ainda não havia nenhum caso de Covid-19 confirmado. Dos 204 municípios, nos quais já havia casos confirmados. Até esta data, a principal cidades afetada pela pandemia foi Belo Horizonte que é a capital do Estado, registrando 915 casos confirmados de Covid-19, seguida de Juiz de Fora, com 214 casos confirmados e Uberlândia, com 225 casos confirmados.

Neste dia, havia 118 óbitos confirmados de Covid-19, sendo Belo Horizonte o município com maior número de óbitos (26), seguidos de Juiz de Fora (21), ${ }^{2}$ Uberlândia (12) ${ }^{3}$. Das vítimas fatais de Covid-19, as pessoas do sexo masculino foram as mais atingidas, com 65 mortes $(54,1 \%)$, enquanto as pessoas do sexo feminino totalizaram que 53 mortes $(44,9 \%)$. Com relação à faixa etária, a maioria dos óbitos ocorreram em pessoas com 60 anos ou mais, 94 (79,7\%), com comorbidades: hipertensão (54), cardiopatia (30), diabetes (28), pneumopatia (20), doença renal (13), neoplasias (12) e obesidade (9). Em $88 \%$ dos óbitos, os pacientes possuem uma ou mais dessas comorbidades (MINAS GERAIS, 2020).

A distribuição dos casos de Covid-19 por sexo apresenta uma diferença significativa, sendo 235 casos $(42,9 \%)$ foram do sexo feminino e 313 casos $(57,1 \%)$ foram do sexo masculino. A faixa etária mais atingida foi de pessoas entre 30 e 39 anos, com 155 casos $(28,3 \%)$, seguido da faixa etária de 40 a 49 com 111 casos (20,3\%). Na faixa etária entre 20 e 59 anos há 413 pessoas infectadas (75,4\%). Os infectados com idade acima de 60 anos são 118 (21,5\%) (MINAS GERAIS, 2020).

Há uma evidência forte de subnotificação dos casos de Covid-19 em Minas Gerais. Como somente os casos hospitalizados graves estão sendo testados, pacientes que vão a óbito em casa ou em enfermarias não estão sendo testados, o número de infectados e o número de óbitos por Covid-19 deve ser bem maiores que os registrados (O TEMPO, 2020).

Para avaliar isso tomamos os casos de Covid-19 notificados no Estado, que até o dia 09/05/2020 eram 113.454. Belo Horizonte, notificou 36.675 casos, Uberlândia 4.688, Contagem 4.427, Betim 3.335 , Ipatinga 2.730, Montes Claros 2,326, Ibirité 2.209 casos, Uberaba 2068, Ribeirão das Neves 1588, Juiz de Fora 1.427 e Nova Lima 1.339 casos notificados (MINAS GERAIS, 2020).

Então, para se entender melhor a gravidade da pandemia, também, pode-se olhar para os dados de hospitalização por Síndrome Respiratória Aguda Grave (SRAG), que até a Semana Epidemiológica (SE) 18 apresentavam um aumento de 513\% em 2020 (1200) em relação a 2019 (7355).

Esta diferença deve ser ainda mais acentuada, porque observando a Figura 2 percebe-se que os dados de 2020, a partir da SE 14 apresentam um decréscimo, não porque o número de casos de SRAG tenham diminuído, pelo contrário, continuaram subindo. É que a maioria dos casos de SRAG deste período ainda estão em investigação e, por isso não podem ser apresentados no gráfico. Esta diferença no incremento de SRAG em 2020 em relação a 2019 deve-se a ocorrência da Covid-19, o que indicaria uma estimativa de 6.155 casos de Covid-19 ao invés de 2.770 casos confirmados no Estado, em 07/05/2020 (Figura 1).

Na Figura 2 se pode observar que a evolução dos casos notificados de Covid-19 em Minas Gerais apresenta 4 fases. A primeira fase, ocorreu a partir da primeira notificação, em 08/02/2020 até $21 / 03 / 2020$, quando ainda era baixa a transmissão e a curva bem achatada, por causa das medidas de isolamento social que estavam sendo adotadas em Minas Gerais, como em todo o país.

\footnotetext{
${ }^{2}$ Confirmado pela Secretaria Municipal de Juiz de Fora, em 11/05/2020

${ }^{3}$ Confirmados pelo Secretaria Municipal de Uberlândia, em 11/05/2020

DOl:http://dx.doi.org/10.14393/Hygeia0054711 $\quad$ Hygeia Edição Especial: Covid-19, Jun./2020 p.243 - 250, pág.245
} 
Figura 1 - Número de hospitalizações por Síndrome Respiratória Aguda Grave (SRAG), segundo semana epidemiológica de início de sintomas, MG, 2019 e 2020

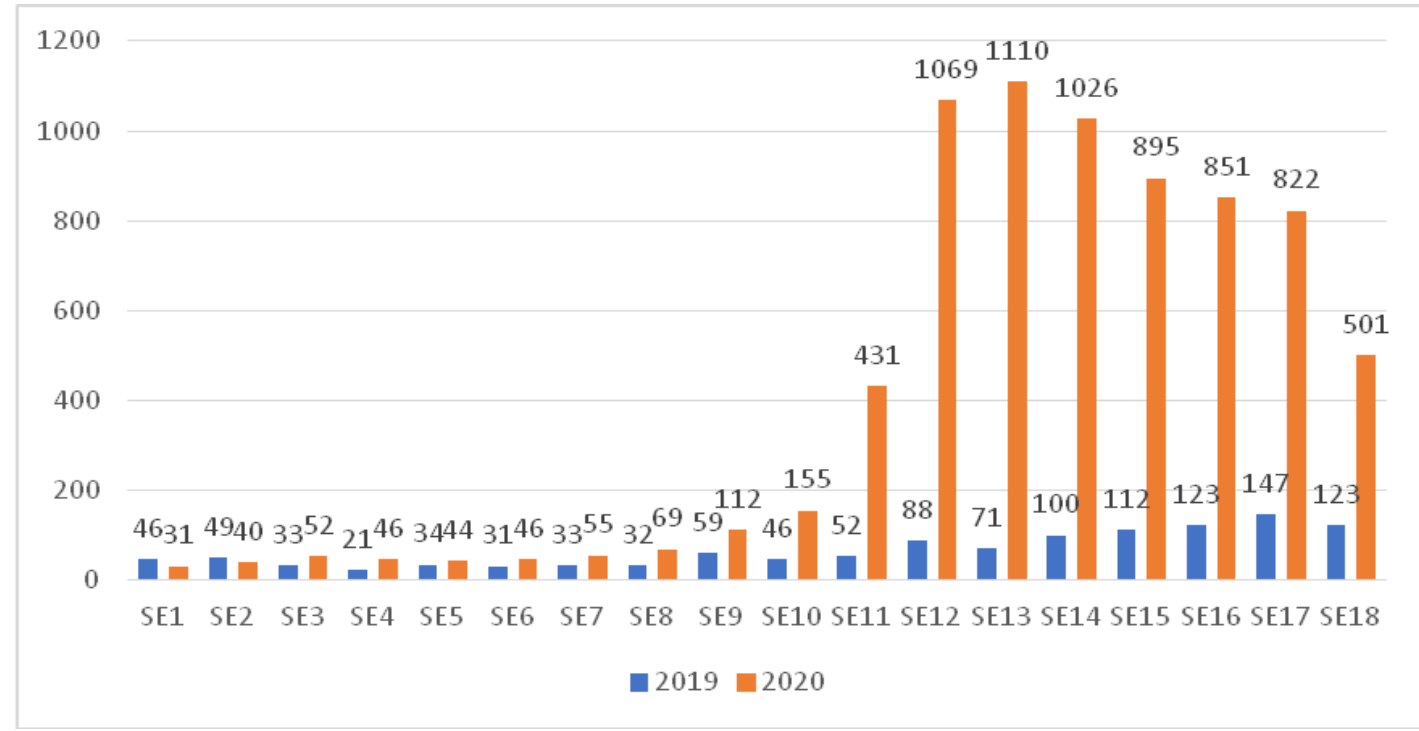

Fonte: MINAS GERAIS (2020), Dados: SIVEP-Gripe. Dados parciais, sujeitos a alterações.

Figura 2 - Minas Gerais: evolução das notificações dos casos Covid-19, até 09/05/2020

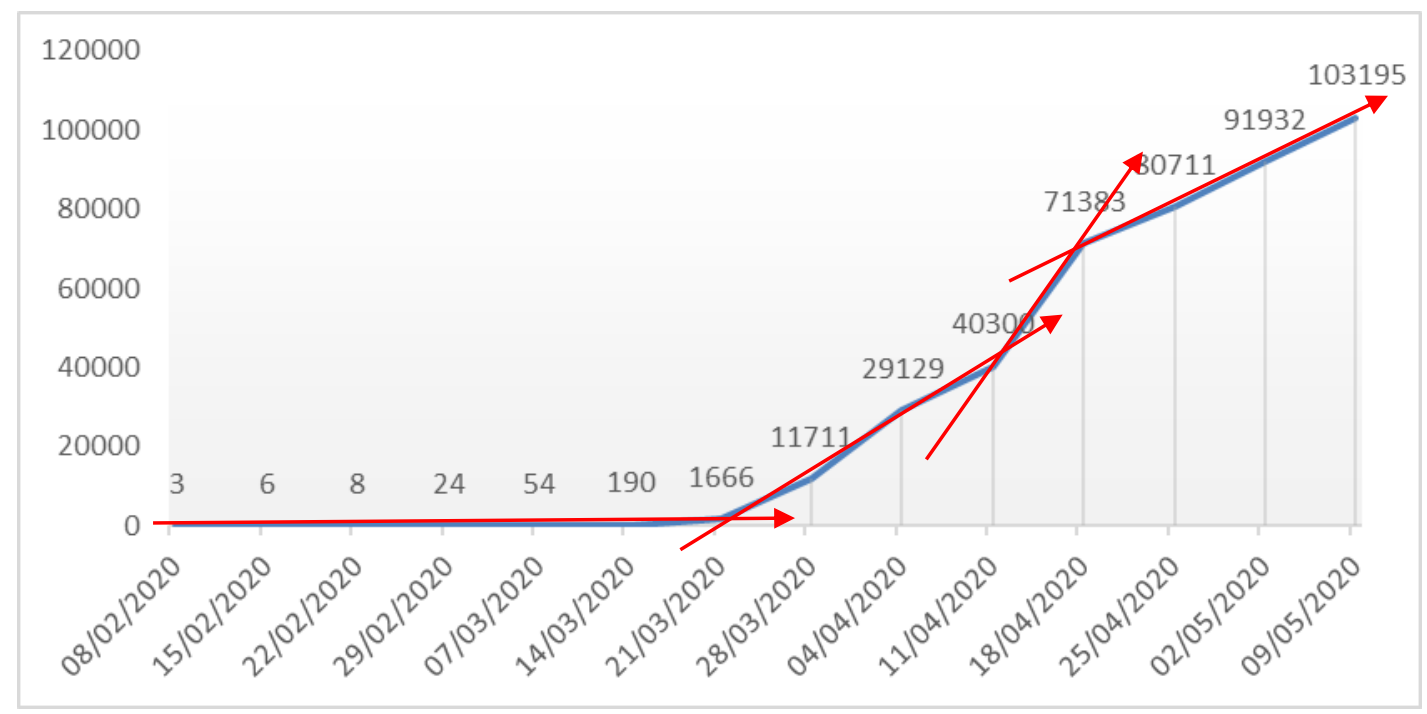

Fonte: MINAS GERAIS (2020), Dados: SIVEP-Gripe. Dados parciais, sujeitos a alterações.

A segunda fase ocorreu entre 21/03/2020 a 11/04/2020, quando o número de notificação de casos de Covid-19 cresceu muito. Isto pode ser explicado pelo flexibilização do isolamento social promovido pelos Estados e Municípios que sofriam pressões de alguns setores da sociedade, principalmente do Presidente da República que avaliaram mal a situação epidemiológica, sugerindo que era preciso abrir o comércio e mandar a população para a rua, em busca da "normalidade", para salvar a economia.

A terceira fase, de 11/04/2020 a 18/04, foi quando a curva de notificação de casos de Covid-19 se inclinou mais, porque a transmissão continuou se acelerando, como reflexo das medidas de flexibilização do isolamento social. 
A quarta fase inicia-se a partir de 18/04/2020, quando observa-se uma pequena redução da inclinação da curva, o que pode não significar uma redução na velocidade da transmissão, mas uma defasagem na publicação dos resultados dos exames. Mas, ainda assim, na última semana de abril, muitos municípios já decretavam flexibilização do isolamento social, abrindo o comércio e liberando a volta ao trabalho em muitas atividades.

\section{DIFUSÃO DA COVID-19 EM MINAS GERAIS}

$\mathrm{O}$ vírus viajou de avião, principalmente com os chineses que saiam da China contaminados. Chegou na Europa em Dezembro, nos EUA em janeiro e chegou no Brasil só em fevereiro. Porque o novo coronavírus chegou primeiro na Europa e nos EUA e só depois chegou ao Brasil? Por causa da maior conectividade internacional, de turismo e de negócios dos países da Europa e dos EUA. Vamos analisar a difusão espacial da Covid-19 a partir de três conceitos fundamentais: conectividade, proximidade e vulnerabilidade social.

Considerando os fluxos do turismo no mundo, vemos que a França recebe 83 milhões de visitantes por ano, seguida dos EUA com 70 milhões de visitantes. A Espanha recebe 70 milhões, a Itália recebe 48 milhões de visitantes e o Brasil recebe por ano cerca de 6 milhões de visitantes. A China recebe 55 milhões de visitantes por ano, em sua maioria turistas da Europa. Isso ajuda explicar porque o novo coronavírus chegou primeiro na Europa, a seguir nos EUA e só depois chegou no Brasil.

No Brasil, o novo coronavírus contaminou primeiro São Paulo, Rio de Janeiro e Fortaleza. Depois foi a vez de Manaus, Belém e Recife. Porque são as capitais brasileiras de maior conectividade internacional, pelos negócios e, principalmente, pelo turismo. Nestas cidades, os bairros de classe média foram os primeiros a receber o vírus, quando os contatos daqueles que viajaram ao exterior foram contaminados. Só depois o vírus se difundiu para os bairros da periferia, infectando os pobres, que vivem em condições de vulnerabilidade social. Este é um momento de grande expetativa, porque a previsão é de que o número de casos de Covid-19 e os óbitos tenha um crescimento exponencial, se não forem tomadas as medidas recomendadas de distanciamento $e$ isolamento social (quarentena).

Nos bairros de periferia, pelo modo de vida precário, as pessoas não conseguem realizar, com frequência, a higiene das mãos, pela dificuldade de acesso à água; o distanciamento social é dificuldade pela precariedade das habitações; e como a maioria das pessoas não têm renda, saem para a rua para trabalhos informais para ganhar hoje o pão que vão comer amanhã. Por essas condições de vulnerabilidade social, o novo cononavírus, deve se difundir rapidamente. Muitas pessoas vão morrer porque o vírus é mortal, mas também pela falta de acesso a um leito de UTI com respirador, quando o sistema de saúde municipal entrar em colapso, quando houver mais doentes que leitos de UTI.

A segunda fase da difusão da pandemia do novo coronavírus foi a contaminação das cidades médias do interior, que somente agora começam a registrar os primeiros casos e óbitos por Covid-19. Isto está acontecendo somente agora, porque essas cidades têm menos conectividade internacional. Como os voos internacionais foram quase todos cancelados e de igual modo os voos domésticos, o novo coronavírus passou a viajar entre as cidades pelas rodovias, de carro ou de ônibus, das capitais para as cidades médias do interior que tinham mais conectividade, pelo fluxo de pessoas e pela proximidade.

A terceira fase da difusão da pandemia, é esperado que em breve se iniciará, seguindo esses mesmos princípios de conectividade e de proximidade, agora chegando às pequenas cidades do interior, que são conectadas às cidades médias, polos regionais. Quanto mais conectadas e mais próximas essas cidades pequenas estiverem destes centros regionais, mais rapidamente serão afetadas. É que as pessoas das cidades pequenas buscam nestas cidades produtos e serviços, principalmente serviços de saúde de maior nível tecnológico. Ao busca saúde nestas cidades podem encontrar o vírus.

A difusão da pandemia no território do Estado de Minas Gerais seguiu o padrão da transmissão da Covid-19 no Brasil. Para demonstrar isso, pode-se olhar para a rede urbana de Minas Gerais, e nela os fluxos da REDIC - IBGE, que definem a hierarquia dos centros urbanos e os fluxos de produtos e serviços, representando a influência das cidades polos sobre as cidades polarizadas (IBGE, 2020).

Pela Figura 3, percebe-se que Belo Horizonte, a capital, polariza todo o Estado, mais principalmente a região central, que Uberlândia e Uberaba polarizam a região do Triângulo e Alto Paranaíba, que DOl:http://dx.doi.org/10.14393/Hygeia0054711 $\quad H y g e i a \quad$ Edição Especial: Covid-19, Jun./2020 p.243 - 250, pág.247 
Juiz de Fora polariza a região sul e zona da mata e que Montes Claros é a cidade que polariza a região norte. Deste modo, é de se esperar a difusão da Covid-19 a partir dessas cidades polos para as pequenas cidades polarizadas em suas redes urbanas, principalmente, porque essas cidades, com exceção de Montes Claros, já estão bastante afetadas pela pandemia.

O princípio da proximidade que influencia o contágio das cidades, também influencia o contágio das pessoas. As cidades que estão flexibilizando o isolamento social para salvar a economia, são aquelas que mais serão impactadas pelo vírus, com maior número de casos e maior número de óbitos por Covid-19. É lógico que manter as pessoas em casa, sem trabalhar, sem comprar afeta o comércio, afeta os negócios, afeta a economia. Isso ocorreu em todo o mundo e os governos socorreram as pessoas e as empresas para manter-se ativas e manter os empregos.

Figura 3 - Minas Gerais: Casos confirmados de Covid-19 e fluxos da REGIC-IBGE, 07/05/2020

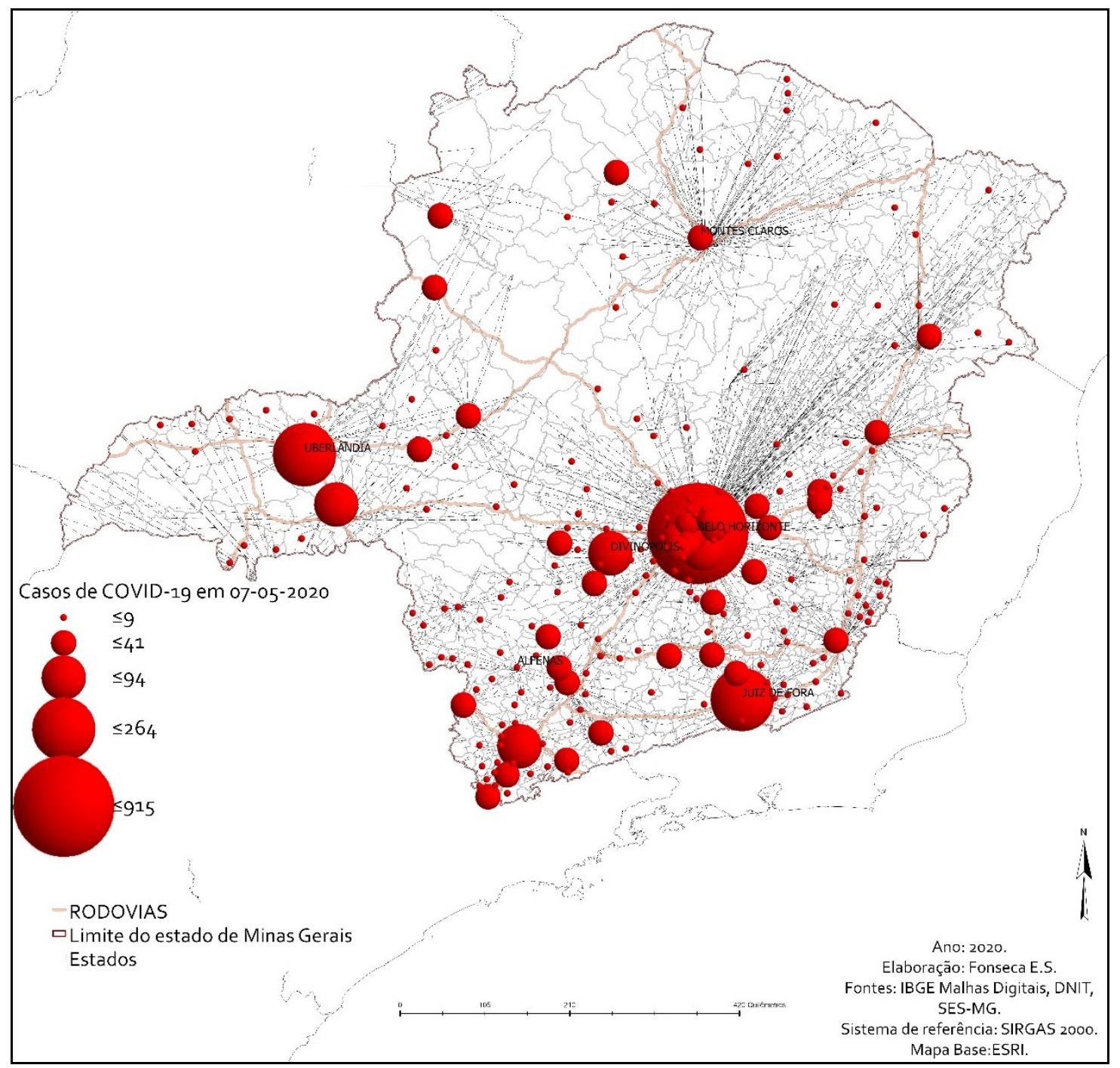

Fonte: MINAS GERAIS (2020), Dados: SIVEP-Gripe. Dados parciais, sujeitos a alterações. 


\section{CONCLUSÕES}

O Estado de Minas Gerais aparece no cenário nacional como um dos menos afetados pela pandemia de Covid-19, pelos dados que a Secretaria de Estado de Saúde de Minas Gerais divulga. Entretanto, essa posição tem sido questionada, tendo em vista que o número de notificação dos casos de Covid19 e o número dos casos de SRAG tem sugerido uma grande subnotificação dos casos confirmados.

Mesmo assim, Belo Horizonte, Juiz de Fora e Uberlândia que são as cidades mais afetadas pela pandemia, ainda não apresentam o mesmo comprometimento do sistema municipal de saúde das grandes metrópoles. Mas, nada garante que o número de casos e de óbitos por Covid-19 se mantenham em níveis controlados. Isso vai depender do grau de comprometimento da população e dos governantes com o distanciamento social e, em determinados casos com o isolamento social (quarentena) mais rígida.

As pequenas cidades do interior, a maioria delas ainda não notificaram casos de Covid-19, mas a expectativa é que essas cidades seja afetadas a partir das cidades polos regionais, Uberlândia e Uberaba no Triângulo e Alto Paranaíba, Juiz de Fora na Zona da Mata e Sul de Minas, Montes Claros no Norte de Minas.

\section{REFERÊNCIAS}

BRASIL. Painel Coronavírus. 2020. Disponível em: <https://covid.saude.gov.br/>. Acesso em: 10 mai. 2020.

BRASIL. Portaria $n^{\circ}$ 454, de 20 de março de 2020. Declara, em todo o território nacional, o estado de transmissão comunitária do coronavírus (covid-19). Ministério da Saúde/Gabinete do Ministro. DOU - Imprensa Nacional. Brasília: 2020. Disponível em: <https://www.sbmfc.org.br/wpcontent/uploads/2020/03/PORTARIA-N\%C2\%BA-454-DE-20-DE-MARC\%CC\%A7O-DE-2020-

PORTARIA-N\%C2\%BA-454-DE-20-DE-MARC\%CC\%A7O-DE-2020-DOU-Imprensa-Nacional.pdf>.

BRASIL. Especial: doença pelo coronavírus 2019. Boletim Epidemiológico, n. 7, 2020.

CFM. Posição do Conselho Federal de Medicina sobre a pandemia de COVID Disponível em: <https://www.sbmfc.org.br/wp-content/uploads/2020/03/POSIC\%CC\%A7A\%CC\%83ODO-CFM-SOBRE-COVID-19-17.03.2020.pdf.pdf.pdf.pdf.pdf>.

IBGE. Regiões de influência das cidades - REGIC. Disponível em $<$ https://www.ibge.gov.br/geociencias/cartas-e-mapas/redes-geograficas/15798-regioes-de-influenciadas-cidades. html $?=\& \mathrm{t}=0$-que-e $>$.

LI, Q.; GUAN, X.; WU, P. et. al. Early Transmission Dynamics in Wuhan, China, of Novel Coronavirus-Infected Pneumonia. The New England Journal of Medicine, vol. 382 no. 13, p. 1199 1207, 2020.

MINAS GERAIS. Cenário em Minas Gerais: Covid-19. Boletim Epidemiológico, n. 19, 2020.

MINAS GERAIS. Confirmação do primeiro caso de Coronavírus (Covid-19) em Minas Gerais. 08/03/2020. SES-MG. Belo Horizonte, 2020. Disponível em: <https://www.saude.mg.gov.br/component/gmg/story/12233-confirmacao-do-primeiro-caso-decoronavirus-covid-19-em-minas-gerais>.

MINAS GERAIS. Boletim Informativo Diário, 09/03/2020. SES-MG. COES Minas Covid-19. Belo Horizonte: 2020.2 Disponível em: <https://saude.mg.gov.br/images/noticias_e_eventos/000_2020/Coronav\%C3\%ADrus/Novo_Boletim_ Coronav\%C3\%ADrus_09-03.pdf>.

O TEMPO. Subnotificação: estudo estima que existam até 21 mil pessoas com Covid-19 em MG. Disponível em em: <https://www.otempo.com.br/cidades/subnotificacao-estudo-estima-queexistam-ate-21-mil-pessoas-com-covid-19-em-mg-1.2342856>. Acesso em 29 mai 2020.

OPAS BRASIL. OMS afirma que COVID-19 é agora caracterizada como pandemia. Disponível em: $<$ https://www.paho.org/bra/index.php?option=com_content\&view=article\&id=6120:oms-afirma-quecovid-19-e-agora-caracterizada-como-pandemia\&Ittemid=812>. Acesso em: 21 abr. 2020. 
REGIANE OLIVEIRA, R. Evolução dos casos de coronavírus no Brasil. EL PAís. São Paulo. 16/04/2020. Disponível em: <https://brasil.elpais.com/ciencia/2020-04-15/evolucao-dos-casos-decoronavirus-no-brasil.html>.

SOUZA, M. V. M. DE; FERREIRA JÚNIOR, D. B. Rede urbana, interações espaciais e a geografia da saúde: análise da trajetória da Covid-19 no estado do Pará. Revista brasileira de geografia econômica, v. 9, n. 11, p. 1-12, 2020. https://doi.org/10.4000/espacoeconomia.13146

STUDDERT, D.M.; HALL, M.A. Disease Control, Civil Liberties, and Mass Testing - Calibrating Restrictions during the Covid-19 Pandemic. The New England Journal of Medicine, vol. 1-3, 2020. Disponível em: https://www.nejm.org/doi/pdf/10.1056/NEJMp2007637?articleTools=true. https://doi.org/10.1056/NEJMp2007637

WU, Z.; MCGOOGAN, J. M. Characteristics of and Important Lessons from the Coronavirus Disease 2019 (COVID-19) Outbreak in China: Summary of a Report of 72314 Cases from the Chinese Center for Disease Control and Prevention. JAMA - Journal of the American Medical Association, v. 323, n. 13, p. 1239-1242, 7 abr. 2020. https://doi.org/10.1001/jama.2020.2648 